University of Texas at El Paso

ScholarWorks@UTEP

\title{
4-2015
}

\section{How to Estimate Expected Shortfall When Probabilities Are Known with Interval or Fuzzy Uncertainty}

Christian Servin

El Paso Community College, cservin@gmail.com

Hung T. Nguyen

New Mexico State University - Main Campus, hunguyen@nmsu.edu

Vladik Kreinovich

The University of Texas at El Paso, vladik@utep.edu

Follow this and additional works at: https://scholarworks.utep.edu/cs_techrep

Part of the Computer Engineering Commons

Comments:

Technical Report: UTEP-CS-15-19a

To appear in Proceedings of the IEEE International Conference on Fuzzy Systems FUZZ-

IEEE'2015, Istanbul, Turkey, August 1-5, 2015.

\section{Recommended Citation}

Servin, Christian; Nguyen, Hung T.; and Kreinovich, Vladik, "How to Estimate Expected Shortfall When Probabilities Are Known with Interval or Fuzzy Uncertainty" (2015). Departmental Technical Reports (CS). 904.

https://scholarworks.utep.edu/cs_techrep/904

This Article is brought to you for free and open access by the Computer Science at ScholarWorks@UTEP. It has been accepted for inclusion in Departmental Technical Reports (CS) by an authorized administrator of ScholarWorks@UTEP. For more information, please contact Iweber@utep.edu. 


\section{How to Estimate Expected Shortfall When Probabilities Are Known with Interval or Fuzzy Uncertainty}

\author{
Christian Servin \\ Information Technology Department \\ El Paso Community College \\ El Paso, TX 79915, USA \\ cservin@gmail.com
}

\author{
Hung T. Nguyen \\ Department of Mathematical Science \\ New Mexico State University \\ Las Cruces, NM 88003, USA \\ and Faculty of Economics \\ Chiang Mai University, Thailand \\ hunguyen@nmsu.edu
}

\author{
Vladik Kreinovich \\ Department of Computer Science \\ University of Texas at El Paso \\ $500 \mathrm{~W}$. University \\ El Paso, Texas 7996, USA \\ vladik@utep.edu
}

\begin{abstract}
To gauge the risk corresponding to a possible disaster, it is important to know both the probability of this disaster and the expected damage caused by such potential disaster ("expected shortfall"). Both these measures of risk are easy to estimate in the ideal case, when we know the exact probabilities of different disaster strengths. In practice, however, we usually only have a partial information about these probabilities: we may have an interval (or, more generally, fuzzy) uncertainty about these probabilities. In this paper, we show how to efficiently estimate the expected shortfall under such interval and/or fuzzy uncertainty.
\end{abstract}

\section{Formulation OF THE PROBlem}

How to gauge risk. In the ideal world, there should be no risk: all engineering designs should be $100 \%$ reliable. To achieve such reliability, civil engineers use the record of historic floods, tsunamis, hurricanes, earthquakes, and other natural disasters to estimate the largest possible strength of such a disaster, and design the buildings, bridges, and other structures so that they can withstand such disasters.

The historic experience shows, however, that there is always a possibility that the disaster strength $S$ exceeds the estimated threshold $s_{0}$ : this was the reason why the hurricane Katrina devastated New Orleans, why in 2011, Fukushima nuclear power station in Japan was destroyed by an unusually high tsunami, etc.

Since we cannot have a threshold $s_{0}$ that would guarantee that the disaster strength never exceeds $s_{0}$, the next best thing is to select a threshold $s_{0}$ for which the probability of exceeding $s_{0}$ does not exceed a given small number $p_{0}$, i.e., for which, with probability $p \stackrel{\text { def }}{=} 1-p_{0} \approx 1$, we have $S \leq s_{0}$.

The choice of the threshold probability $p_{0}$ depends on the situation. For example:

- $\quad$ for manned space flights, NASA selected $p_{0}=10^{-3}$; smaller values were not feasible because of high uncertainty associated with space flights;

- $\quad$ on the other extreme, for reliability of a cell forming a computer memory, we need $p_{0} \ll 10^{-9}$, because otherwise, if we allow $p_{0} \gg 10^{-9}$, at least one of the billions of cells will always go wrong.
In addition to knowing the threshold $s_{0}$, it is also desirable to also know how much damage will come, on average, if this threshold is exceeded. For each possible value $S$ of the corresponding disaster strength, we can estimate the corresponding damage $X$; the stronger the disaster, the larger the damage. Let $x_{p}$ denote the damage corresponding to the threshold value $s_{0}$; then, the condition $S \geq s_{0}$ is equivalent to $X \geq x_{p}$.

In these terms, the probability that the disaster strength exceeds the threshold $s_{0}$ is equal to $\operatorname{Prob}\left(X>x_{p}\right)$. In addition to this probability, it is desirable to also know the conditional expectation of the damage under the condition that the disaster strength exceeds the threshold $x_{p}$, i.e., the value

$$
\mathrm{ES}_{p} \stackrel{\text { def }}{=} E\left[X \mid X \geq x_{p}\right]
$$

The corresponding conditional expectation is known as expected shortfall. These two values:

- $\quad$ the threshold $x_{p}$, and

- the expected shortfall $\mathrm{ES}_{p}$,

is how we gauge the risk.

Similar two measures are used in finance to describe the risk that an investment would result in a big loss; see, e.g., [4].

How to estimate the expected shortfall in the ideal case, when we know the probability distribution describing damage. In the ideal case, we know the probability distribution that describes possible values of the damage $X$. A usual way to describe a probability distribution is by describing its cumulative distribution function (cdf) $F(x) \stackrel{\text { def }}{=} \operatorname{Prob}(X \leq x)$; see, e.g., [6].

In terms of cdf, the probability of exceeding the threshold value $x_{p}$ is simply equal to $1-F\left(x_{p}\right)$. Thus, we have $1-F\left(x_{p}\right)=p_{0}$ and hence, $F\left(x_{p}\right)=1-p_{0}=p$. For each probability $p$, the value $x_{p}$ for which $F\left(x_{p}\right)=p$ is known as the $p$-th quantile. For example:

- $\quad$ for $p=0.5$, we get the median; 
- $\quad$ for $p=0.25$ and $p=0.75$, we get quartiles,

etc. In mathematical terms, the function that maps $p$ to $x_{p}$ is an inverse function to the cdf $F(x)$.

The conditional expectation can then be computed as the ratio

$$
\mathrm{ES}_{p}=\frac{\int_{x_{p}}^{\infty} x d F(x)}{1-p} .
$$

In practice, we only have partial information about the probabilities. In practice, we rarely know the exact values of all the probabilities, we only have partial information about these probabilities. This may mean that, instead of the exact values $F(x)$ corresponding to different values $x$, we only know an interval $[\underline{F}(x), \bar{F}(x)]$ that contains the actual (unknown) value $F(x)$. Such situation when, for each $d$, we only know the corresponding intervals, is known as a probability box or, for short, a $p$-box; see, e.g., [1], [2].

Even more generally, for each $x$, we may have several intervals $[\underline{F}(x), \bar{F}(x)]$ corresponding to different degrees of certainty $\alpha \in[0,1]$, i.e., in effect, a fuzzy number; see, e.g., [3], [5], [7].

How to gauge risk under such an uncertainty? For different distributions $F(x) \in[\underline{F}(x), \bar{F}(x)]$ within a given p-box, we get different values of quantiles $x_{p}$ for which $F\left(x_{p}\right)=p$. One can easily check that:

- $\quad$ the smallest value $x_{p}$ corresponds to the largest values $\bar{F}(x)$ of the cdf; while

- $\quad$ the largest value $x_{p}$ corresponds to the smallest values $\underline{F}(x)$ of the cdf.

Thus, possible values of the quantile $x_{p}$ form an interval $\left[\underline{x}_{p}, \bar{x}_{p}\right]$ in which $\bar{F}\left(\underline{x}_{p}\right)=\underline{F}\left(\bar{x}_{p}\right)=p$.

Such quantile intervals are often useful when we perform computations with p-boxes; see, e.g., [1], [2].

We can use this idea to handle the case when we have a fuzzy-valued function $\mathbf{F}(x)$, if we take into account the known fact that for all possible computation $y=f\left(x_{1}, \ldots, x_{n}\right)$ with fuzzy numbers, the alpha-cut

$$
{ }^{\alpha} \mathbf{y} \stackrel{\text { def }}{=}\{y: \mu(y) \geq \alpha\}
$$

of the result is equal to the range

$$
\begin{gathered}
f\left({ }^{\alpha} \mathbf{x}_{1}, \ldots,{ }^{\alpha} \mathbf{x}_{n}\right)= \\
\left\{f\left(x_{1}, \ldots, x_{n}\right): x_{1} \in{ }^{\alpha} \mathbf{x}_{1}, \ldots, x_{n} \in{ }^{\alpha} \mathbf{x}_{n}(\alpha)\right\}
\end{gathered}
$$

of the values $f\left(x_{1}, \ldots, x_{n}\right)$ when each $x_{i}$ belongs to the corresponding $\alpha$-cut ${ }^{\alpha} \mathbf{x}_{i}=\left\{x_{i}: \mu_{i}\left(x_{i}\right) \geq \alpha\right\}$; see, e.g., [3], [5].

Thus, to find the $\alpha$-cut of the quantile $x_{p}$, it is sufficient to compute the interval $\left[\underline{x}_{p}, \bar{x}_{p}\right]$ in situation when each $F(x)$ belongs to the corresponding $\alpha$-cut of the fuzzy number $\mathbf{F}(x)$.

Such straightforward computation is possible because the dependence of the probability $p=F\left(x_{p}\right)$ on the unknown function $F(x)$ is monotonic (namely, increasing), and that each cdf $F(x)$ is also an increasing function. So, if we increase the values of the function $F(x)$, then for newly increased function $\widetilde{F}(x)$, we will have $\widetilde{F}\left(x_{p}\right)>F\left(x_{p}\right)=p$. Thus, to find the value $\widetilde{x}_{p}$ for which $\widetilde{F}\left(\bar{x}_{p}\right)=p$, we need to decrease the value $x_{p}: \widetilde{x}_{p}<x_{p}$.

So, to find the range of possible values $\left[\underline{x}_{p}, \bar{x}_{p}\right]$ of the quantile $x_{p}$, we do not need to enumerate all possible functions $F(x)$ from the p-box $[\underline{F}(x), \bar{F}(x)]$ - because of monotonicity, we know that:

- the smallest value $\underline{x}_{p}$ of the quantile $x_{p}$ is attained when the cdf $F(x)$ attains its largest possible value, i.e., when $F(x)=\bar{F}(x)$ for all $x$; and

- the largest value $\bar{x}_{p}$ of the quantile $x_{p}$ is attained when the cdf $F(x)$ attains is smallest possible value, i.e., when $F(x)=\underline{F}(x)$ for all $x$.

For expected shortfall, different probability distributions from the p-box, in general, lead to different values. We would like to find the range of possible values of the expected shortfall. Estimating this range, however, is not a very straightforward task: when we increase $F(x)$, it is not a priori clear whether the corresponding integral increases or decreases. Thus, we cannot immediately come up with a simple expression for the range of the expected shortfall.

It is therefore necessary to come up with efficient algorithms for estimating the range of the expected shortfall under p-box (interval) uncertainty. Similarly, for the fuzzy case, we need to be able to transform the fuzzy-valued $\operatorname{cdf} \mathbf{F}(x)$ into a fuzzy value for the resulting expected shortfall.

What we do in this paper. In this paper, we provide the efficient algorithms for computing the expected shortfall under interval (p-box) and fuzzy uncertainty.

To be more precise, we produce an algorithm for computing the expected shortfall under interval (p-box) uncertainty. Based on what we mentioned earlier about fuzzy computations, to find the $\alpha$-cut of the expected shortfall, it is sufficient to compute the range of possible values of $\mathrm{ES}_{p}$ of the expected shortfall in situation when each $F(x)$ belongs to the corresponding $\alpha$-cut of the fuzzy number $\mathbf{F}(x)$. In other words, from the algorithmic viewpoint, the problem of computing the expected shortfall under fuzzy uncertainty can be indeed reduced to the case of interval (p-box) uncertainty.

\section{ANALysis OF THE PROBlem}

Let us find an equivalent expression for $\mathrm{ES}_{p}$. To find the range of possible values of expected shortfall $\mathrm{ES}_{p}$, let us find an equivalent expression for $\mathrm{ES}_{p}$ that would simplify the computation of this range.

According to formula (1), we have

$$
\mathrm{ES}_{p}=\frac{1}{1-p} \cdot I,
$$

where

$$
I \stackrel{\text { def }}{=} \int_{x_{p}}^{\infty} x d F(x) .
$$

Thus: 
- the expected shortfall $\mathrm{ES}_{p}$ attains its smallest possible value $\underline{\mathrm{ES}}_{p}$ when the integral $I$ attains its smallest possible value $\underline{I}$, and

- the expected shortfall $\mathrm{ES}_{p}$ attains its largest possible value $\overline{\mathrm{ES}}_{p}$ when the integral $I$ attains its largest possible value $\bar{I}$.

The integral $I$ has an infinite upper bound. This integral can be thus represented as a limit of integrals $I_{T}$ with a finite upper bound $T$ when $T \rightarrow \infty$ :

$$
I=\lim _{T \rightarrow \infty} I_{T}
$$

where

$$
I_{T} \stackrel{\text { def }}{=} \int_{x_{p}}^{T} x d F(x) .
$$

Thus, for very large $T$, we have $I \approx I_{T}$.

The integral $I_{T}$ can be integrated by part:

$$
\begin{gathered}
I_{T}=\left.x \cdot F(x)\right|_{x_{p}} ^{T}-\int_{x_{p}}^{T} F(x) d x= \\
T \cdot F(T)-x_{p} \cdot F\left(x_{p}\right)-\int_{x_{p}}^{T} F(x) d x .
\end{gathered}
$$

For large $T$, we have $F(T)$ practically equal to

$$
\lim _{T \rightarrow \infty} F(T)=1
$$

so $T \cdot F(T)=T$ and

$$
I_{T}=T-x_{p} \cdot F\left(x_{p}\right)-\int_{x_{p}}^{T} F(x) d x .
$$

By definition of a quantile $x_{p}$, we have $F\left(x_{p}\right)=p$, so

$$
I_{T}=T-x_{p} \cdot p-\int_{x_{p}}^{T} F(x) d x .
$$

\section{When does the integral $I_{T}$ attain its largest and smallest possible values? According to our analysis:}

- the expected shortfall $\mathrm{ES}_{p}$ attains its largest possible value when the value of the integral $I_{T}$ (corresponding to a very large value $T$ ) is the largest possible, and

- the expected shortfall $\mathrm{ES}_{p}$ attains its smallest possible value when the value of the integral $I_{T}$ (corresponding to a very large value $T$ ) is the smallest possible.

Let us thus analyze when the integral $I_{T}$ attains its largest and smallest possible values.

When does the integral $I_{T}$ attain its largest possible value? Let us start with the largest possible value. Different cdfs $F(x)$ from the given p-box result, in general, in different values of the integral $I_{T}$. Let $x_{p}^{\max }$ be the value corresponding to the cdf $F^{\max }(x)$ for which this integral is the largest possible. This means, in particular, that among all cdfs $F(x)$ with the same value of the $p$-th quantile $x_{p}^{\max }$ (i.e., for which $F\left(x_{p}^{\max }\right)=p$ ), this particular cdf $F^{\max }(x)$ leads to the largest possible value of the integral $I_{T}$.
One can easily see, from the expression (9), that the integral $I_{T}$ is a decreasing function of the values $F(x)$. Thus, this integral is the largest when all the values $F(x)$ are the smallest. What limitations on the values $F(x)$ do we have?

- We have a limitation $\underline{F}(x) \leq F(x) \leq \bar{F}(x)$ coming from the fact that we only consider cdfs from a given p-box $[\underline{F}(x), \bar{F}(x)]$.

- We also have a limitation $F(x) \geq F\left(x_{p}\right)=p$, which, for values $x \geq x_{p}$, comes from the requirement $F\left(x_{p}\right)=p$ and from the fact that each cdf is an increasing function of $x$.

These constraints $\underline{F}(x) \leq F(x) \leq \bar{F}(x)$ and $F(x) \geq p$ can be equivalently described by a single constraint

$$
\max (\underline{F}(x), p) \leq F(x) \leq \bar{F}(x) .
$$

Thus, the smallest possible values of $F(x)$ correspond to

$$
F(x)=\max (\underline{F}(x), p) .
$$

When $\underline{F}(x) \geq p$, we have

$$
\max (\underline{F}(x), p)=\underline{F}(x)
$$

and hence $F(x)=\underline{F}(x)$. As we described earlier, the equality $\underline{F}(x)=p$ is equivalent to $x=\bar{x}_{p}$, thus the condition $\underline{F}(x) \geq p$ is equivalent to $x \geq \bar{x}_{p}$.

On the other hand, when $\underline{F}(x)<p$, i.e., equivalently, when $x<\bar{x}_{p}$, we have $F(x)=p$. Thus,

$$
\begin{gathered}
\int_{x_{p}^{\max }}^{T} F(x) d x=\int_{x_{p}^{\max }}^{\bar{x}_{p}} p d x+\int_{\bar{x}_{p}}^{T} \underline{F}(x) d x= \\
\left(\bar{x}_{p}-x_{p}^{\max }\right) \cdot p+\int_{\bar{x}_{p}}^{T} \underline{F}(x) d x .
\end{gathered}
$$

Thus, the expression (9) takes the form

$$
I_{T}=T-x_{p}^{\max } \cdot p-\left(\bar{x}_{p}-x_{p}^{\max }\right) \cdot p-\int_{\bar{x}_{p}}^{T} \underline{F}(x) d x .
$$

The two terms $x_{p}^{\max } \cdot p$ and $\left(\bar{x}_{p}-x_{p}^{\max }\right) \cdot p$ can be easily combined into a single term $\bar{x}_{p} \cdot p$, so

$$
I_{T}=T-\bar{x}_{p} \cdot p-\int_{\bar{x}_{p}}^{T} \underline{F}(x) d x .
$$

Since $\bar{x}_{p}$ is the quantile corresponding to the lower endpoint $\underline{F}(x)$ of the p-box, we can therefore conclude that the expression (14) is the value of the integral $I_{T}$ corresponding to $F(x)=\underline{F}(x)$.

Thus, the largest value of the integral $I_{T}$ - and hence, of the expected shortfall - is attained when $F(x)=\underline{F}(x)$.

When does the integral $I_{T}$ attain its smallest possible value? Let us now consider the smallest possible value. Let $x_{p}^{\mathrm{min}}$ be the value corresponding to the cdf $F^{\min }(x)$ for which this integral is the largest possible. This means, in particular, that among all cdfs $F(x)$ with the same value of the $p$-th quantile $x_{p}^{\min }$ (i.e., for which $F\left(x_{p}^{\min }\right)=p$ ), this particular cdf $F^{\min }(x)$ leads to the smallest possible value of the integral $I_{T}$. 
Since the integral $I_{T}$ is a decreasing function of the values $F(x)$, this integral is the smallest when all the values $F(x)$ are the largest. Under the limitations (10), the largest possible values are $F(x)=\bar{F}(x)$.

Thus, the smallest value of the integral $I_{T}$ - and hence, of the expected shortfall $\mathrm{ES}_{p}$ - is attained when $F(x)=\bar{F}(x)$.

\section{Resulting Algorithm: CASE of Interval UNCERTAINTY}

Problem: reminder. We want to find the range $\left[\underline{\mathrm{ES}}_{p}, \overline{\mathrm{ES}}_{p}\right]$ of possible values of the expected shortfall $\mathrm{ES}_{p}$ when cdf $F(x)$ is in the given p-box $[\underline{F}(x), \bar{F}(x)]$.

Conclusion. The above analysis leads to the following conclusion:

- The largest possible value $\overline{\mathrm{ES}}_{p}$ of the expected shortfall $\mathrm{ES}_{p}$ is attained when $F(x)=\underline{F}(x)$ for all $x$.

- The smallest possible value $\overline{\mathrm{ES}}_{p}$ of the expected shortfall $\mathrm{ES}_{p}$ is attained when $F(x)=\bar{F}(x)$ for all $x$.

Thus, we arrive at the following algorithm:

\section{Resulting algorithm.}

- First, we compute the expected shortfall $\mathrm{ES}_{p}$ corresponding to $F(x)=\underline{F}(x)$, as the ratio

$$
\frac{1}{1-p} \cdot \int_{\bar{x}_{p}}^{\infty} x d \underline{F}(x),
$$

where $\bar{x}_{p} \stackrel{\text { def }}{=}(\underline{F})^{-1}(p)$. This ratio is the upper endpoint $\overline{\mathrm{ES}}_{p}$ of the desired interval $\left[\underline{\mathrm{ES}}_{p}, \overline{\mathrm{ES}}_{p}\right]$.

- Then, we compute the expected shortfall $\mathrm{ES}_{p}$ corresponding to $F(x)=\bar{F}(x)$, as the ratio

$$
\frac{1}{1-p} \cdot \int_{\underline{x}_{p}}^{\infty} x d \bar{F}(x),
$$

where $\underline{x}_{p} \stackrel{\text { def }}{=}(\bar{F})^{-1}(p)$. This ratio is the lower endpoint $\underline{\mathrm{ES}}_{p}$ of the desired interval $\left[\underline{\mathrm{ES}}_{p}, \overline{\mathrm{ES}}_{p}\right]$.

\section{Resulting Algorithm: CAse of FuZZy UNCERTAINTY}

Problem: reminder. We have a fuzzy-valued cdf $\mathbf{F}(x)$. In other words, for every real number $x$, we know a fuzzy number $\mathbf{F}(x)$ that describes the probability $\operatorname{Prob}(X \leq x)$.

We are also given a probability $p$. We want to compute the corresponding fuzzy shortfall $\mathbf{E S}_{p}$.

Idea. To compute the desired fuzzy number $\mathbf{E S}_{p}$, we compute, for each value $\alpha \in[0,1]$, the corresponding $\alpha$-cut ${ }^{\alpha} \mathbf{E S}_{p}$.

Of course, in practice, we can only perform computations with finitely many values $\alpha$, so usually, we choose the threshold values $\alpha$ for degrees of certainty in accordance with the accuracy with which we can realistically determine these degrees - e.g., for values $\alpha=0, \alpha=0.1, \alpha=0.2, \ldots$, $\alpha=0.9$, and $\alpha=1.0$.
As we have mentioned earlier, the interval forming this $\alpha$-cut can be computed by solving the interval-uncertainty problem in which we use the $\alpha$-cuts of the inputs.

In our case, the inputs are the values of the fuzzy cdf $\mathbf{F}(x)$. Thus, to compute the desired $\alpha$-cut ${ }^{\alpha} \mathbf{E S}_{p}$, we can solve the corresponding interval-uncertainty problem in which the inputs are the $\alpha$-cuts ${ }^{\alpha} \mathbf{F}(x)$ of the fuzzy cdf.

We already know how to solve the corresponding intervaluncertainty problem, so we arrive at the following fuzzy-case algorithm.

Resulting algorithm. We start with the $\alpha$-cuts

$$
{ }^{\alpha} \mathbf{F}(x)=\left[{ }^{\alpha} \underline{F}(x),{ }^{\alpha} \bar{F}(x)\right]
$$

corresponding to the values of the fuzzy cdf $\mathbf{F}(x)$. Our objective is to compute the $\alpha$-cuts ${ }^{\alpha} \mathbf{E S}_{p}$ of the expected shortfall $\mathbf{E S}_{p}$.

For that purpose, for each $\alpha$, we perform the following computations:

- First, we compute the expected shortfall $\mathrm{ES}_{p}$ corresponding to the $\operatorname{cdf}{ }^{\alpha} \underline{F}(x)$, as the ratio

$$
\frac{1}{1-p} \cdot \int_{\alpha \bar{x}_{p}}^{\infty} x d^{\alpha} \underline{F}(x),
$$

where ${ }^{\alpha} \bar{x}_{p} \stackrel{\text { def }}{=}\left({ }^{\alpha} \underline{F}\right)^{-1}(p)$. This ratio is the lower endpoint ${ }^{\alpha} \underline{\mathrm{ES}}_{p}$ of the desired $\alpha$-cut

$$
{ }^{\alpha} \mathbf{E S}_{p}=\left[{ }^{\alpha} \underline{\mathrm{ES}}_{p},{ }^{\alpha} \overline{\mathrm{ES}}_{p}\right] .
$$

- Then, we compute the expected shortfall $\mathrm{ES}_{p}$ corresponding to the cdf ${ }^{\alpha} \bar{F}(x)$, as the ratio

$$
\frac{1}{1-p} \cdot \int_{\alpha_{\underline{x}_{p}}}^{\infty} x d^{\alpha} \bar{F}(x),
$$

where ${ }^{\alpha} \underline{x}_{p} \stackrel{\text { def }}{=}\left({ }^{\alpha} \bar{F}\right)^{-1}(p)$. This ratio is the upper endpoint ${ }^{\alpha} \overline{\mathrm{ES}}_{p}$ of the desired $\alpha$-cut

$$
{ }^{\alpha} \mathbf{E S}_{p}=\left[{ }^{\alpha} \underline{\mathrm{ES}}_{p},{ }^{\alpha} \overline{\mathrm{ES}}_{p}\right]
$$

\section{ACKNOWLEDGMENT}

This work was supported in part by the Faculty of Economics of Chiang Mai University and by the National Science Foundation grants HRD-0734825 and HRD-1242122 (CyberShARE Center of Excellence) and DUE-0926721.

The authors are thankful to Scott Ferson and Paul Embrechts for valuable discussions, and to the anonymous referees for important suggestions.

\section{REFERENCES}

[1] S. Ferson, Risk Assessment with Uncertainty Numbers: RiskCalc, CRC Press, Boca Raton, Florida, 2002.

[2] S. Ferson, V. Kreinovich, W. Oberkampf, and L. Ginzburg, Experimental Uncertainty Estimation and Statistics for Data Having Interval Uncertainty, Sandia National Laboratories, Report SAND2007-0939, 2007.

[3] G. J. Klir and B. Yuan, Fuzzy Sets and Fuzzy Logic: Theory and Applications, Prentice Hall, Upper Saddle River, New Jersey, 1995. 
[4] A. J. McNeil, R. Frey, and P. Embrechts, Quantitative Risk Management: Concepts, Techniques, Tools, Princeton University Press, Princeton, New Jersey, 2010.

[5] H. T. Nguyen and E. A. Walker, A First Course in Fuzzy Logic, Chapman and Hall/CRC, Boca Raton, Florida, 2006.

[6] D. J. Sheskin, Handbook of Parametric and Nonparametric Statistical Procedures, Chapman \& Hall/CRC, Boca Raton, Florida, 2011.

[7] L. A. Zadeh, "Fuzzy sets", Information and Control, 1965, Vol. 8, pp. $338-353$. 